\title{
Impact of kidney donation on changing in physical, emotional, and socioeconomic status
}

\author{
${ }^{1}$ Department of Internal Medicine-Nephrology, Keimyung University Dongsan Medical Center, Daegu, Korea \\ ${ }^{2}$ Department of Internal Medicine-Nephrology, Dongguk University Ilsan Hospital, Goyang, Korea \\ ${ }^{3}$ Department of Internal Medicine-Nephrology, Ewha Womans University Seoul Hospital, Seoul, Korea \\ ${ }^{4}$ Department of Biostatistics, Korea University College of Medicine, Seoul, Korea \\ ${ }^{5}$ Department of Internal Medicine-Nephrology, Seoul National University Hospital, Seoul, Korea
}

Yaerim Kim ${ }^{1}$, Jang Wook Lee ${ }^{2}$, Eunjung Kang ${ }^{3}$, Jina Park ${ }^{4}$, Sehoon Park ${ }^{5}$, Yong Chul Kim ${ }^{5}$, Yon Su Kim ${ }^{5}$, Hajeong Lee ${ }^{5}$

Background: Kidney donation induces not only a physical burden but also an emotional, socioeconomic burden in living kidney donors. Composed to the substantial interest for major clinical outcomes such as mortality and end-stage kidney disease, subjective conditions including physical, emotional, and socioeconomic status were disregarded for exploring.

Methods: A total of 429 donors were recruited for the survey in two tertiary hospitals in Korea between February and November 2020. The survey was conducted by divided into pre-donation and post-donation. The survey was composed of baseline characteristics, questions for quality of life, including subjective health score, 36-Item Short Form Survey, Patient Health Questionnaire-9, and socioeconomic status. We used McNemar's test and paired t-test for comparing the characteristics between the pre- and post-donation.

Results: There were 130 and 299 donors who answered pre- and post-donation surveillance, respectively. The main reason to decide donation was the willingness to recipients' health and happiness (59.1\%) or families' benefit and happiness (52.3\%). Also, the main concern to hesitate to donate was physical problems such as the potential risk of surgical complications (22.6\%) and kidney failure (20.8\%). The subjective health score was significantly decreased from 100 to 36.3 after donation. All categories in SF-36 showed worse change, and the score for depressive mood was also significantly increased after donation. Nevertheless, most donors (84.4\%) answered they would donate again if they can go back to before donation. Most employed donors experienced vacation for more than 2 months include unpaid vacation. Payment for kidney donation was performed by donors (40.8\%), recipients (35.4\%), and divided (17.5\%). There were $25.8 \%$ of donors who unaware of the refund system after donation.

Conclusions: Although donation raised multifarious burdens, most donors had a willingness to donate for altruistic reasons. Multiplicative supports comprise of social and medical aspects would be essential to encourage affirmative donation with considering both aspects of donors and recipients.

Corresponding author: Hajeong Lee

E-mail: mdhjlee@gmail.com

(c) The Korean Society for Transplantation

This is an Open Access article distributed under the terms of the Creative Commons Attribution Non-Commercial License (http://creativecommons.org/licenses/by-nc/4.0/) which permits unrestricted non-commercial use, distribution, and reproduction in any medium, provided the original work is properly cited. 